\title{
A relation between the right triangle and circular tori with constant mean curvature in the unit 3 -sphere
}

\author{
ABDÊNAGO BARROS \\ Departamento de Matemática-UFC, B1 914, Campus do Pici, 60455-760 Fortaleza, CE, Brasil \\ Manuscript received on May 30, 2003; accepted for publication on June 14, 2004; \\ presented by MANFREDO DO CARMO*
}

\begin{abstract}
In this note we will show that the inverse image under the stereographic projection of a circular torus of revolution in the 3-dimensional euclidean space has constant mean curvature in the unit 3 -sphere if and only if their radii are the catet and the hypotenuse of an appropriate right triangle.
\end{abstract}

Key words: Flat torus, constant mean curvature, circular tori, stereographic projection.

\section{INTRODUCTION}

We will denote by $T(r, a)$ the standard circular torus of revolution in $\mathbb{R}^{3}$ obtained from the circle $\Gamma$ in the $x z-$ plane centered at $(r, 0,0)$ with radius $a<r$, i.e.

$$
T(r, a)=\left\{(x, y, z) \in \mathbb{R}^{3}:\left(\sqrt{x^{2}+y^{2}}-r\right)^{2}+z^{2}=a^{2}\right\} .
$$

Now let $\rho: \mathbb{S}^{3} \backslash\{n\} \rightarrow \mathbb{R}^{3}$ be the stereographic projection of the Euclidean sphere $\mathbb{S}^{3}=\{x \in$ $\left.\mathbb{R}^{4}:|x|^{2}=1\right\}$, where $n=(0,0,0,1)$ is its north pole. The inverse image of a circular torus in $\mathbb{R}^{3}$ under the stereographic projection will be called a circular torus in $\mathbb{S}^{3}$. We would like to know when circular tori in $\mathbb{R}^{3}$ comes from constant mean curvature circular tori in $\mathbb{S}^{3}$ under the stereographic projection. A circular torus in $\mathbb{S}^{3}$ meant that it is obtained from a revolution of a circle in $\mathbb{S}^{3}$ under a rigid motion. A general $T(r, a)$ will not satisfy the above requirement. For instance, it was proved by Montiel and Ros (Montiel and Ros 1981) that a compact embedded surface $S$ with constant mean curvature contained in an open hemisphere of $\mathbb{S}^{3}$ must be a round sphere. Hence for $T(r, a)$ contained inside or outside of the unit ball $B(1) \subset \mathbb{R}^{3}, \rho^{-1}(T(r, a))$ will be contained in an open hemisphere of $\mathbb{S}^{3}$ and can not have constant mean curvature. Then among

*Member Academia Brasileira de Ciências

E-mail: abbarros@mat.ufc.br

AMS Classification: Primary 53A05, 53A10; Secondary 53A30. 
all tori $T(r, a)$ which intercept the inside and the outside of the unit ball $B(1)$ we will describe those which have the desired property. We will show that to construct such a torus we take an arbitrary point $P(\alpha)=(\cos \alpha, 0, \sin \alpha)$ on the unit circle of the $x z-$ plane, $0<\alpha<\pi / 2$, draw its tangent until it meets the $x$ axis at the point $Q(\alpha)=(\sec \alpha, 0,0)$ which will be the center of the circle $\Gamma$ whereas its radius will be $a=\tan \alpha$, i.e. the torus $T(\sec \alpha, \tan \alpha)$ will satisfy the previous requirement. We note if $O$ denotes the origin of $\mathbb{R}^{3}$ then the triangle $O P Q$ is a right triangle. This description will yield that the Clifford torus is associated to a right triangle with two equal sides. More precisely, our aim in this note is to present a proof of the following fact:

THEOREM 1. Let $T^{2} \subset \mathbb{S}^{3}$ be a circular torus of constant mean curvature. Then

$$
T^{2}=\rho^{-1}(T(\sec \alpha, \tan \alpha))=S^{1}(\cos \alpha) \times S^{1}(\sin \alpha) .
$$

Moreover, the mean curvature of $T^{2}$ is given by $\bar{H}=\frac{\left(\tan ^{2} \alpha-1\right)}{2 \tan \alpha}$.

\section{PRELIMINARIES}

For an immersion $f: M \rightarrow \bar{M}$ between Riemannian manifolds we will denote by $d s_{f}^{2}$ the induced metric on $M$ by $f$. Now let $M^{n}, M_{1}^{m}$ and $M_{2}^{m}$ be Riemannian manifolds, where the superscript denote the dimension of the manifold. Consider $\psi: M^{n} \rightarrow M_{1}^{m}$ be an immersion, $\rho: M_{1}^{m} \rightarrow M_{2}^{m}$ a conformal mapping and set $\varphi=\rho \circ \psi$. Let $\phi: M \rightarrow \mathbb{R}$ be a function verifying $d s_{\varphi}^{2}=e^{2 \phi} d s_{\psi}^{2}$. If $\bar{k}_{i}$ and $k_{i}$ denote the principal curvatures of $\psi$ and $\varphi=\rho \circ \psi$, respectively, then we get

$$
k_{i}=e^{-\phi}\left(\bar{k}_{i}-\frac{\partial \phi}{\partial \xi}\right),
$$

where $\xi$ is a unit normal vector field to $\psi(M)$, see for instance (Abe 1982) or (Willmore 1982). At first we will recall the following known lemma of which we sketch the proof.

Lemma 1. Let $\psi=\left(\psi_{1}, \psi_{2}, \psi_{3}, \psi_{4}\right): M^{2} \rightarrow \mathbb{S}^{3} \backslash\{n\}$ be an immersion of a surface $M^{2}$, set $\varphi=\rho \circ \psi$ and suppose $d s_{\varphi}^{2}=e^{2 \phi} d s_{\psi}^{2}$. Then we get

$$
k_{i}=e^{-\phi}\left(\bar{k}_{i}-g\right) \text {, }
$$

where $g=\langle\nu, \varphi\rangle$ denotes the support function on $M^{2} \subset \mathbb{R}^{3}$.

Proof. If we put $\psi=\psi\left(u_{1}, u_{2}\right)$ then a direct computation gives

$$
\left\langle\frac{\partial \varphi}{\partial u_{i}}, \frac{\partial \varphi}{\partial u_{j}}\right\rangle=\lambda^{2}\left\langle\frac{\partial \psi}{\partial u_{i}}, \frac{\partial \psi}{\partial u_{j}}\right\rangle,
$$

where $\lambda=\left(1-\psi_{4}\right)^{-1}=\frac{1+|\varphi|^{2}}{2}$. So we can write $d s_{\varphi}^{2}=e^{2 \phi} d s_{\psi}^{2}$ with $e^{\phi}=\frac{1+|\varphi|^{2}}{2}$. Thus if $v$ denotes a unit normal vector field to $\varphi\left(M^{2}\right)$ then $v=e^{-\phi} \xi$, where $\xi$ stands for a unit normal vector field to $\psi\left(M^{2}\right)$. Hence we have from (1)

$$
k_{i}=e^{-\phi} \bar{k}_{i}-\frac{\partial \phi}{\partial v}=e^{-\phi}\left(\bar{k}_{i}-\langle v, \varphi\rangle\right)=e^{-\phi}\left(\bar{k}_{i}-g\right),
$$

as we wished to prove. 


\section{PROOF OF THE THEOREM}

Proof. First we note that the circle $\Gamma=\left\{(x, 0, z) \in \mathbb{R}^{3}:(x-r)^{2}+z^{2}=a^{2}\right\}$ can be parametrized by the map $\gamma:[0,2 \pi] \rightarrow \mathbb{R}^{3}$ defined by

$$
\gamma(t)=\left(\frac{r^{2}-a^{2}}{r-a \sin t}, 0, \frac{a \sqrt{r^{2}-a^{2}} \cos t}{r-a \sin t}\right) .
$$

In fact, it is enough to check that

$$
\left(\frac{r^{2}-a^{2}}{r-a \sin t}-r\right)^{2}+\left(\frac{a \sqrt{r^{2}-a^{2}} \cos t}{r-a \sin t}\right)^{2}=a^{2}
$$

Representing by $R_{\theta}$ a rotation on $\mathbb{R}^{3}$ around the $z-$ axis, we see that $R_{\theta}(\gamma(t))$ is a circular torus $T(r, a)$ if $\gamma$ is a parametrization of the circle $\Gamma$ given above. We put now $\sigma=\sqrt{r^{2}-a^{2}}$, $\theta=r u_{1} / \sigma^{2}$ and $t=r u_{2} / a \sigma$. We note that such a choice implies $0 \leq u_{1} \leq\left(2 \pi \sigma^{2}\right) / r$ and $0 \leq u_{2} \leq(2 \pi a \sigma) / r$. Let us call $R_{\theta}(\gamma(t))$ of $\varphi\left(u_{1}, u_{2}\right)$, i.e.

$$
\varphi\left(u_{1}, u_{2}\right)=\sigma(r-a \sin t)^{-1}(\sigma \cos \theta, \sigma \sin \theta, a \cos t) .
$$

Hence we have

$$
e^{\phi}=\frac{1+|\varphi|^{2}}{2}=\frac{q(t)}{2(r-a \sin t)},
$$

where $q(t)=a\left(\sigma^{2}-1\right) \sin t+r\left(\sigma^{2}+1\right)$. Now a straightforward computation yields

$$
\left\{\begin{aligned}
\frac{\partial \varphi}{\partial u_{1}} & =\frac{r}{(r-a \sin t)}(-\sin \theta, \cos \theta, 0) \\
\frac{\partial \varphi}{\partial u_{2}} & =\frac{r}{(r-a \sin t)^{2}}(\sigma \cos t \cos \theta, \sigma \cos t \sin \theta, a-r \sin t)
\end{aligned}\right.
$$

From that we derive that $\varphi$ is a conformal parametrization of $T(r, a)$ satisfying

$$
\left\langle\frac{\partial \varphi}{\partial u_{i}}, \frac{\partial \varphi}{\partial u_{j}}\right\rangle=\frac{r^{2} \delta_{i j}}{(r-a \sin t)^{2}}
$$

Moreover, a unit vector field normal to $\varphi$ is given as follows:

$$
v\left(u_{1}, u_{2}\right)=-\frac{1}{(r-a \sin t)}((a-r \sin t) \cos \theta,(a-r \sin t) \sin \theta,-\sigma \cos t) .
$$

Therefore we conclude that

$$
g=\frac{\sigma^{2} \sin t}{(r-a \sin t)}
$$


On the other hand a new computation gives us

$$
\left\{\begin{aligned}
\frac{\partial v}{\partial u_{1}} & =-\frac{(a-r \sin t)}{\sigma^{2}} \frac{\partial \varphi}{\partial u_{1}} \\
\frac{\partial v}{\partial u_{2}} & =\frac{1}{a} \frac{\partial \varphi}{\partial u_{2}}
\end{aligned}\right.
$$

From this we have $k_{1}=\frac{(a-r \sin t)}{\left(r^{2}-a^{2}\right)}$ and $k_{2}=-\frac{1}{a}$. Taking into account (5), (7) and (8) we conclude from Lemma 1 that

$$
\bar{H}=\frac{1}{4 a \sigma^{2}}\left(r a\left(\sigma^{2}-1\right) \sin t+\left(\sigma^{2}+1\right)\left(2 a^{2}-r^{2}\right)\right) .
$$

Now we have that $\bar{H}$ is constant if and only if $\sigma^{2}=1$. Moreover, $\sigma^{2}=1$ yields $\bar{H}=\frac{1}{2 a}\left(a^{2}-1\right)$. Since $a<r$ we put $a=r \sin \alpha, r=\sec \alpha$ and this completes the proof of the theorem.

We point out that $\bar{H}=0$ if and only if $a=1$ and $r=\sqrt{2}$ which corresponds to the right triangle with two equal sides.

\section{THE WILLMORE MEASURE ON $T(r, a)$}

In this section we will present a simple way to compute $\int_{T(r, a)} H^{2} d A$ by using the parametrization of $T(a, r)$ given by (4). We observe that if $d A$ denotes the element of area of $T(r, a)$ then its Willmore measure is given by

$$
\left(H^{2}-K\right) d A=\frac{r^{4}}{4 a^{2} \sigma^{4}} d u_{1} d u_{2} .
$$

Hence, using Gauss-Bonnet theorem, we easily conclude that

$$
\int_{T(r, a)} H^{2} d A=\frac{r^{4}}{4 a^{2} \sigma^{4}} \int_{0}^{\frac{2 \pi a \sigma}{r}} \int_{0}^{\frac{2 \pi \sigma^{2}}{r}} d u_{1} d u_{2}=\frac{r^{2}}{a \sqrt{r^{2}-a^{2}}} \pi^{2}
$$

Therefore the family of tori $T(\sqrt{2} a, a)$, which corresponds to the family of right triangles with two equal sides, yields the minimum for $\int_{T(r, a)} H^{2} d A$ among all circular tori. Moreover, from (9) its value is (see also Willmore 1982)

$$
\int_{T(\sqrt{2} a, a)} H^{2} d A=2 \pi^{2} .
$$

Since $a<r$, if we choose $\alpha$ such that $\sin \alpha=\frac{a}{r}$, we conclude from (9) the following corollary.

COROLlARY 1. Given a circular torus $T(r, a) \subset \mathbb{R}^{3}$ we have a circular torus $T(\sec \alpha, \tan \alpha) \subset \mathbb{R}^{3}$ such that $\int_{T(r, a)} H^{2} d A=\int_{T(\sec \alpha, \tan \alpha)} H_{\alpha}^{2} d A_{\alpha}$. In other words, the family of circular tori with constant mean curvature in $\mathbb{S}^{3}$ cover all values of $\int_{T(r, a)} H^{2} d A$. 


\section{CONCLUDING REMARKS}

We point out that Theorem 2 of K. Nomizu and B. Smyth (Nomizu and Smyth 1969) guarantees that a flat torus of constant mean curvature in $\mathbb{S}^{3}$ is isometric to a product of circles. Then $\rho^{-1} T(a, r)$ is flat if and only if it has constant mean curvature. We notice if we set $\psi=\rho^{-1} \varphi$ where $\varphi$ was given by (4) then we have

$$
\psi\left(u_{1}, u_{2}\right)=\frac{1}{q(t)}\left(2 \sigma^{2} \cos \theta, 2 \sigma^{2} \sin \theta, 2 a \sigma \cos t, r\left(\sigma^{2}-1\right)+a\left(\sigma^{2}+1\right) \sin t\right),
$$

where $q(t)=a\left(\sigma^{2}-1\right) \sin t+r\left(\sigma^{2}+1\right)$, (see(5)). Hence by using (3), (5), (6) and putting $z=u_{1}+i u_{2}$ we conclude that

$$
d s_{\psi}^{2}=e^{-2 \phi} d s_{\varphi}^{2}=\frac{4 r^{2}}{q^{2}(t)}|d z|^{2} .
$$

According to our theorem the metric $d s_{\psi}^{2}$ is flat if and only if $\rho^{-1} T(r, a)$ has constant mean curvature in $\mathbb{S}^{3}$. In this case we have

$$
\psi\left(u_{1}, u_{2}\right)=\frac{1}{\sqrt{a^{2}+1}}(\cos \theta, \sin \theta, a \cos t, a \sin t),
$$

i.e. $\rho^{-1} T(r, a)$ is isometric to the product of circles $S^{1}\left(\frac{1}{\sqrt{a^{2}+1}}\right) \times S^{1}\left(\frac{a}{\sqrt{a^{2}+1}}\right)$. We note that this yields $\cos \alpha=\frac{1}{\sqrt{a^{2}+1}}$ and $\sin \alpha=\frac{a}{\sqrt{a^{2}+1}}$, i.e. $\rho\left(S^{1}(\cos \alpha) \times S^{1}(\sin \alpha)\right)=T(\sec \alpha, \tan \alpha)$.

\section{ACKNOWLEDGMENTS}

This work was partially supported by FINEP-Brazil.

\section{RESUMO}

Neste artigo mostraremos que a imagem inversa pela projeção estereográfica de um toro circular de revolução no espaço euclidiano de dimensão 3 tem curvatura média constante se e somente se os seus raios são o cateto e a hipotenusa de um triângulo retângulo apropriado.

Palavras-chave: Toro plano, Curvatura média constante, Toro circular, Projeção estereográfica.

\section{REFERENCES}

ABE N. 1982. On generalized total curvature and conformal mappings. Hiroshima Math J 12: 203-207.

Montiel S AND Ros A. 1981. Compact hypersurfaces: The Alexandrov theorem for higher order mean curvatures, A symposium in honor of Manfredo do Carmo, Edited by B. Lawson and K. Tenenblat, Pitman Monographs 52: 279-296.

Nomizu K AND Smyth B. 1969. A formula of Simons' type and hypersurfaces with constant mean curvature. J Diff Geom 3: 367-377.

WiLlmore T. 1982. Total curvature in Riemannian geometry, Ellis Horwood limited, 168 pp. 